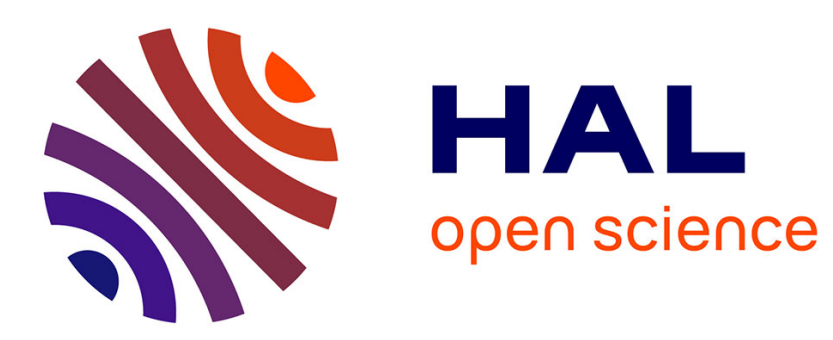

\title{
Modelling errors calculation adapted to rainfall - runoff model user expectations and discharge data uncertainties
}

\author{
Audrey Douinot, Hélène Roux, Denis Dartus
}

\section{To cite this version:}

Audrey Douinot, Hélène Roux, Denis Dartus. Modelling errors calculation adapted to rainfall - runoff model user expectations and discharge data uncertainties. Environmental Modelling and Software, 2017, 90, pp.157-166. 10.1016/j.envsoft.2017.01.007 . hal-01457058

\section{HAL Id: hal-01457058 \\ https://hal.science/hal-01457058}

Submitted on 9 Feb 2017

HAL is a multi-disciplinary open access archive for the deposit and dissemination of scientific research documents, whether they are published or not. The documents may come from teaching and research institutions in France or abroad, or from public or private research centers.
L'archive ouverte pluridisciplinaire HAL, est destinée au dépôt et à la diffusion de documents scientifiques de niveau recherche, publiés ou non, émanant des établissements d'enseignement et de recherche français ou étrangers, des laboratoires publics ou privés. 


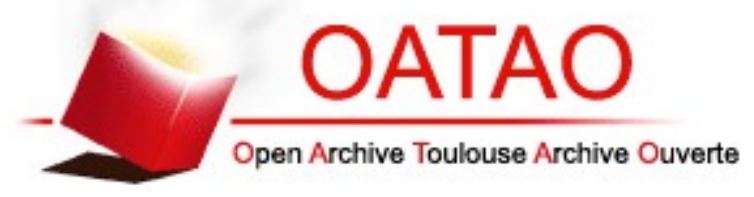

\section{Open Archive Toulouse Archive Ouverte (OATAO)}

OATAO is an open access repository that collects the work of Toulouse researchers and makes it freely available over the web where possible.

This is an author-deposited version published in: http://oatao.univ-toulouse.fr/ Eprints ID: 17481

To link to this article : DOI: 10.1016/j.envsoft.2017.01.007

URL : http://dx.doi.org/10.1016/j.envsoft.2017.01.007

To cite this version: Douinot, Audrey and Roux, Hélène and Dartus, Denis Modelling errors calculation adapted to rainfall - runoff model user expectations and discharge data uncertainties. (2017) Environmental Modelling and Software, vol. 90. pp. 157-166. ISSN 1364-8152

Any correspondence concerning this service should be sent to the repository administrator: staff-oatao@listes-diff.inp-toulouse.fr 


\title{
Modelling errors calculation adapted to rainfall - Runoff model user expectations and discharge data uncertainties
}

\author{
Audrey Douinot*, Hélène Roux, Denis Dartus \\ Institut de Mécanique des Fluides de Toulouse (IMFT), Université de Toulouse, CNRS-INPT-UPS, Toulouse France
}

Keywords:

Uncertainty propagation

Hydrological modelling

Calibration

Evaluation

Discharge uncertainty

Error isolines

\begin{abstract}
A B S T R A C T
A novel objective function for rainfall-runoff model calibration, named Discharge Envelop Catching (DEC), is proposed. DEC meets the objectives of: i) taking into account uncertainty of discharge observations, ii) enabling the end-user to define an acceptable uncertainty, that best fits his needs, for each part of the simulated hydrograph. A calibration methodology based on DEC is demonstrated on MARINE, an existing hydrological model dedicated to flash floods. Calibration results of state-of-the-art objective functions are benchmarked against the proposed objective function. The demonstration highlights the usefulness of the DEC objective function in identifying the strengths and weaknesses of a model in reproducing hydrological processes. These results emphasize the added value of considering uncertainty of discharge observations during calibration and of refining the measure of model error according to the objectives of the hydrological model.
\end{abstract}

\section{Introduction}

An objective function converts the outputs of a rainfall-runoff model into a single likelihood measure, according to discharge measurements. This likelihood measure plays a key role, as it controls the model assessment and calibration. As such it provides a comparison basis for models or scenarios. An objective function must provide a meaningful criterion, representative of the errors occurring in the prediction time series. Ideally the objective function must make a distinction between the observed errors coming from data uncertainties and the modelling errors coming from model limitations and parameter uncertainties. Defining such a metric is hard, as model outputs obviously depend on the input data and the observed discharge quality.

The uncertainty of the forcing data (rainfall/snowfall, soil moisture, etc.) is in general not measurable (Villarini and Krajewski (2010); Kirstetter et al. (2010)) whereas discharge uncertainties can be accurately quantified (McMillan and Westerberg, 2015; Coxon et al. (2015); Le Coz et al. (2014)). This makes it possible to integrate uncertainty of the discharge observationst into an objective function. However the classical functions, such as the NashSutcliffe efficiency (NSE, Nash and Sutcliffe, 1970), or the Kling-

\footnotetext{
* Corresponding author.

E-mail address: audrey.douinot@imft.fr (A. Douinot).
}

Gupta-Efficiency (KGE, Gupta et al., 2009), are based on the difference between the model outputs and the observed discharge, without considering the discharge uncertainty. This can result in the overfitting of a model prediction to uncertain discharge observations.

Some modifications in different calibration approaches are found in the literature in order to integrate uncertainty of the discharge observations. Croke (2007) modified the NSE by weighting the residual vector according to the accuracy of observed discharge measurement. The metric thus emphasizes the prediction of a well known observed discharge at the expense of the observed discharge with high uncertainty. This is especially problematic in the context of flood modelling, where extreme flood discharges are generally marred with high uncertainty. Calibration methods based on Bayesian approach (Kuczera (1983); Engeland and Gottschalk, 2002, Kavetski et al., 2006), formalize an error model, considering among others discharge uncertainty. Formalizations of different type of errors, such as input uncertainty or model uncertainty are based on strong assumptions that require validation, which is not always possible. In the end, the calibration results depend on the definition of the error model. Liu et al. (2009) proposed a calibration method using a "limits-of-acceptability" approach. A parametrization is either accepted or rejected. The limit of acceptability is fixed according to discharge uncertainty. The method is convenient to assess the likelihood of a parameter set for a model, but it does not provide information on the 
relevance of the model.

The aim of the paper is to provide an objective function: i) taking into account uncertainty of the discharge observations; ii) adapting the calibration to user expectations and model assumptions; iii) providing a meaningful score which can be interpreted to assess the relevance of the model.

Section 2 presents the rationale of the paper. It discusses the state of the art of objective functions in the field of hydrologic models, with a focus on the model calibration issue. The proposed objective function, called Discharge Envelop Catching efficiency, is defined in Section 3 and evaluated against three other objectives functions in Section 4. Finally, calibration results are presented and discussed in Section 5.

\section{Background and motivation}

We begin the section introducing the mathematical concepts used throughout the paper.

\subsection{Mathematical notation and symbols}

We adopt the fomulation of Vrugt and Sadegh (2013) of model calibration and evaluation issues: "Consider a discrete vector of measurements $\hat{Y}=\left\{\hat{y}_{1}, \ldots, \hat{y}_{n}\right\}$, observed at times $t=\{1, \ldots, n\}$ that summarizes the response of an environmental system $\mathrm{F}$ to forcing variables $\hat{U}=\left\{\hat{u}_{1}, \ldots, \hat{\mathrm{u}}_{n}\right\}$. Let $Y=\left\{y_{1}, \ldots, y_{\mathrm{n}}\right\}$ the corresponding predictions from a dynamic (non linear) model $f$, with parameter values $\theta$,

$Y(\theta)=f\left(x_{0}, \theta, \widehat{U}\right)$

where $x 0$ is the initial state of the system at $t=0$." The residual vector defines the difference between actual and model-simulated system behaviours:

$E(\theta)=\widehat{Y}-Y(\theta)=\left\{e_{1}(\theta), \ldots, e_{n}(\theta)\right\}$

The error model $\mathrm{F}$ that allows for residual vector transformation defines the modelling error vector:

$\varepsilon(\theta)=F[\widehat{Y}-Y(\theta)]=\left\{\varepsilon_{1}(\theta), \ldots, \varepsilon_{n}(\theta)\right\}$

A function $\mathrm{G}$ is used to map the modelling error vector into a metric called likelihood measure. The combination of $F$ and $G$ is the objective function.

Calibration aims to find the values of $\left[\theta \in \Theta \in \Re^{\mathrm{d}}\right.$ ] that provide the best likelihood measure. As the optimal parameter set may not be unique and several candidates may minimized equally the objective function, the calibration process faces model equifinality (Beven and Binley, 1992; Beven, 2006). Choosing a way of selecting or weighting behavioural parameter sets according to likelihood measure corresponds to the last step of a calibration methodology.

We now consider the fact that forcing variables $\hat{U}$, initial state $x_{0}$ and observed discharges $\hat{Y}$ are uncertain measurements and denote $\sigma_{\hat{U}}, \sigma_{X 0}, \sigma_{\hat{Y}}$ the vectors quantifying those uncertainties. Forcing variables and initial state uncertainties affect model predictions and modify equation ( 1 ):

$Y^{\prime}(\theta)=f\left(x_{0}\left|\sigma_{x_{0}}, \theta, \widehat{U}\right| \sigma_{\widehat{U}}\right)$

where $Y^{\prime}(\theta)$ is the model prediction with respect to input uncertainties. Similarly, the observed discharge uncertainties modify equation (3):
$\varepsilon(\theta)=F\left[\widehat{Y} \mid \sigma_{\widehat{Y}}-Y(\theta)\right]=\left\{\varepsilon_{1}(\theta), \ldots, \varepsilon_{n}(\theta)\right\}$

This paper focuses on equation (5) and proposes an error model $F$ that allows for benchmarking a model prediction vector $Y(\theta)$ against uncertain observations $\left(\hat{Y}, \sigma_{\hat{Y}}\right)$. The choice of the optimal function $\mathrm{G}$ which maps the modelling error vector into a metric is also discussed.

\subsection{Adapting the likelihood measure to the model}

As said before, the primary goal of calibration is finding parameter sets that best mimic the observed discharge. The role of the objective function is to define the most appropriate likelihood measure to accurately assess the success of the model to reproduce the hydrological behavior of a catchment system.

In the literature, performance models are usually assessed using statistic scores such as linear correlation, mean, variance or indexes widespread in the hydrology community such as NSE, RMSE or Kling-Gupta-Efficiency (KGE, Gupta et al. (2009)). The use of those scores as conventional likelihood measures is supposed to facilitate model comparison. However, as pointed out by Seibert (2001) or Schaefli and Gupta (2007), a score may reflect poorly the goodnessof-fit of a model, even when established by hydrologists. As an example, a NSE score of 0,6 could equally mean good or poor fit depending on data quality and on the studied catchment. Moussa (2010) and Schaefli et al. (2005) also highlighted the limitations of the NSE for flood event modelling assessment, showing that considering the high value of standard deviation of discharge time series, the residuals might be high and still lead to a good score, due to the NSE definition.

Schaefli and Gupta (2007) suggested to take into account model assumptions and user expectations into the objective function. They defined the benchmark efficiency (BE):

$B E=1-\frac{\sum_{i=1}^{n}\left(y_{i}-\widehat{y_{i}}\right)^{2}}{\sum_{i=1}^{n}\left(\widehat{y_{i}}-y_{b i}\right)^{2}}$

where $y_{b i}$ is called the benchmark discharge model at time i. The model reference is no more the observed discharge mean as in NSE, but a benchmark model defined as admissible by the hydrologist. The BE definition implies a meaningful score according to what is expected from the model.

All the objective functions seen so far choose to minimize the sum of squared residuals as the calibration objective. As noticed by Beven and Binley (2014), this is not without implication. The combination of all residuals within a single value actually hides the underlying assumption that this score represents at best all the residuals. Assuming that the sum of squared residual is the best representation has two important implications:

- the same importance is attached to all residual values, whatever their position along the hydrograph. Yet, absolute errors during high flows or low flows may not be interpreted the same by hydrologist. This issue could be avoided by weighting residual vector as in mNSE (Croke, 2007) or calculating the sum of squared relative errors;

- among the residual distribution, the mean represents the best index to minimize. As residuals are most commonly correlated, heteroscedastic and have non-Gaussian distributions (Schoups and Vrugt (2010)), the relevance of this choice is not certain. Moreover, the mean of the residual distribution is mainly 
affected by residuals observed during low flows, which are highly correlated and over-represented.

The NSE and other likelihood measures that consider the sum of squared residual provide a basis for model assessment. However the underlying assumptions are not consistent with residual vector properties. The interplay of the above implications, balancing each other, result in a global adaptation of the measure for calibrating large data time series based on wrong criteria, which can contribute to misleading results.

\subsection{Taking into account the uncertainty of the discharge observations}

Considering the residual vector as an evaluation of model error assumes that the discharge observations are the exact reflect of the hydrological behavior of the catchment. However, discharge time series are successively extracted from stage measurements and stage-discharge rating curve conversions and, consequently, may contain highly uncertain values. In other words, model evaluation based on the residual vector $E(\theta)$ is limited by the uncertainty on the discharge data. In parallel, recent contributions (McMillan and Westerberg, 2015; Coxon et al. (2015); Le Coz et al. (2014)). improved discharge uncertainty quantification. As an example, Le Coz et al. (2014) used knowledge of the hydraulic control of the rating-curves and statistical methods to provide an individual quantification of gauging uncertainty. Taking into account discharge uncertainties in an objective function enables to better define the discharge benchmark, making it possible to extract modelling error from the residual vector.

Several approaches accounting for the uncertainty of the discharge observations in the calibration methods are proposed in the literature. (Kavetski et al., 2003; Kuczera et al. (2006)). use a Bayesian framework. They represent the observed discharge as a formal probability density function, the function being determined either according to the rating curve uncertainty (Thyer et al. (2009)) or by adding another parameter to define the discharge uncertainty model (Huard and Mailhot, 2008). Defining error models for input uncertainty as well as model uncertainty, the bayesian approach aims to calibrate at the same time the parameters of the hydrological model and those of the error models. Although the calibration is comprehensive, as it tends to consider all the approximations done, it suffers from the lack of benchmark for error models. It may also result in overparametrization, increasing the complexity of calibration. It can be noticed also that the Bayesian approach assumes that the modelling errors are uncorrelated, which is plausible for inputs, for instance, but less so for the model. Indeed, model uncertainty cannot be smaller than that of the observation dataset used to calibrate the model.

Discharge uncertainty has also been incorporated into objective functions as weights of the residual vectors (Croke (2007); PeñaArancibia et al., 2015). For instance Croke (2007) has modified the NSE, introducing the mNSE which uses weights $\gamma_{i}$ inferred from the discharge uncertainty:

$$
m N S E=1-\frac{\sum_{i=1}^{n} \gamma_{i}\left(y_{i}-\widehat{y}_{i}\right)^{2}}{\sum_{i=1}^{n} \gamma_{i}\left(\widehat{y}_{i}-\bar{y}\right)^{2}} \text { with } \gamma_{i}=\frac{1}{\widehat{y}_{i}^{95 t h}-\widehat{y}_{i}^{5 t h}}
$$

where $\hat{y}_{i}^{95 t h}$ and $\hat{y}_{i}^{5 \text { th }}$ are the 95 th percentile and the 5 th percentile values of the probability density function of the discharge flow at time $i$. The discharge uncertainty is considered as an assessment of the discharge measurements quality but does not clarify the values of the discharge observations. This calibration enforces the model to be accurate when data is accurately known, whereas it allows for large modelling errors where data is uncertain, which makes it unsuited for flood forecasting, for instance.

Another take on the issue is proposed by Liu et al. (2009), with the limits of acceptability approach. The simulation set $[Y(\theta), \theta \in \Theta$ $\left.\in \Re^{\mathrm{d}}\right]$ is separated between behavioural and non behavioural simulations according to observation error (Hornberger and Spear, 1981). The selection is done by setting a minimum percentage of prediction time steps that must be included in the confidence interval of discharge measurements. Then, a weighted score is attributed to each simulation time step. The score decreases linearly with distance to observed discharge, tending to zero in the boundaries of the confidence interval. When the value of the simulated discharge falls beyond those limits, the score is uniformly set to zero. A first limitation lies in the subjective choice of the percentage threshold used to separate behavioural from non behavioural simulations. If the bounds of the confidence interval of the discharge measurement are set to the xth percentile and the $(100-x)^{\text {th }}$ percentile values of the distribution function, $a(100-2 x)$ value might logically be used as a percentage threshold. However, this choice assumes an ideal model devoid of modelling errors. Thus, the threshold might need to be adjusted according to the ability of the model to mimic the discharge observations. Also, as mentioned by Liu et al. (2009), time steps not included in the confidence interval might be the ones with the highest "hydrological value". As the weighting method gives equal weights for those time steps and for the ones lying exactly on the confidence interval bounds, small or large distances from the confidence interval limits do not affect the return value of the objective function. In other words, the score does not assess how far the prediction is from the observed discharge.

Objective functions presented above propose different approaches including the uncertainty of discharge observations into model assessment. However, they do not consider additionally model specifics and expectations from which tolerated modelling errors might be deduced. It is actually important to distinguish what we can require from the hydrological prediction according to the uncertainty of discharge observations and what we can require from it according to model assumptions and data input uncertainty. The first point refers to the fact that the objective of the exact reproduction of the observed discharge values is misleading. The second point refers to the fact that it is not because a discharge is really accurately measured, that we could expect the same accuracy in prediction. The objective of this work is to take advantage of the hydrologist expertise and of the uncertainty of the discharge observations to adapt the measure of error of rainfall-runoff models to the end-user expectations. The subsequent novel objective function is called Discharge Envelop Catching (DEC) and presented hereafter.

\section{The discharge envelop catching (DEC) objective function}

\subsection{Definition of the error model}

We assume that the uncertainty of the discharge observations is available. For any time i, the discharge is defined by a probability density function from which the mean value $\hat{y}_{i}$, standard deviation $\sigma_{\hat{y} i}$ or any percentile $\hat{y}_{i}^{x t h}$ can be extracted. A confidence interval of the discharge observations can be defined.

Instead of looking for the exact reproduction of discharge measurement, we aim at minimizing the distance between the simulated discharge and the confidence interval of observed discharge. Moreover, the objective function will define for each evaluation point a range of acceptable distances according to user 
expectations. The error model F, used in the DEC, extracts from the residual vector $\hat{Y}-Y(\theta)$, a standard measure $\varepsilon_{\text {mod, } i}$ of the distance between the prediction and the confidence interval:

$\varepsilon_{\text {mod }, i}=\frac{d_{i}}{\sigma_{\text {mod }, i}}$

where $d_{i}$ corresponds to the discharge distance $\left[\mathrm{m}^{3} . \mathrm{s}^{-1}\right]$ between the model prediction at time $i\left(y_{i}\right)$ and the confidence interval of discharge measurements, knowing that $y_{i}$ is located outside the bounds. $\sigma_{\text {mod,i, }}$ called modelling distance bounds at time $i$, corresponds to the distance range $\left[\mathrm{m}^{3} . \mathrm{s}^{-1}\right]$ that is considered acceptable by the user at time $i$.

Setting the distance range value for each time $i$, the user can specify how the model will be forced throughout calibration to get closer to the confidence interval. The modelling error is relevant given that: if $\varepsilon_{\text {mod, } i} \leq 1$, the model prediction $y_{i}$ is acceptable, whereas if $\varepsilon_{\text {mod, } i}>1$, user expectations are not respected by $y_{i}$. Finally, the $\Omega_{\bmod }=\left(\sigma_{\text {mod, } i}\right)$ vector defines a region of acceptability for discharge prediction enclosing the confidence interval of discharge observations.

The objective function, combining the evaluation of distances and the explanation of the user expectations, results in a vector of modelling error $E_{\text {mod }}=\left(\varepsilon_{\text {mod, } i}\right)$ whose statistical properties are representative of the overall prediction error of the model.

We consider the 90 percentile of the distribution, $E_{\text {mod }}^{90 t}$, as the likelihood measure:

$D E C=E_{\text {mod }}{ }^{90 t h}$

The calibration metric $E_{\text {mod }}^{\text {9ot }}$ will tend to standardize modelling error distribution, to prioritize minimization of the largest modelling errors, while limiting the issue due to the correlated nature of the modelling errors.

\subsection{A graphical representation of an objective function: the error isolines}

Error isolines are a graphical representation related to an objective function. An error isoline is composed of prediction points exerting an equal impact on the objective function. Two predictions at different time will have the same impact on the likelihood measure if they are located on the same error isoline.

Error isolines may uncover the assumptions underlying a given objective function. As such, they offer a way to compare several objective functions. Fig. 1 displays the error isolines of the NSE, Liu et al. (2009), Croke (2007) and DEC objective functions:

- the top left window (a) displays error isolines of the NSE objective function. They also map BE or any objective function using the sum of squared residuals to reduce the residual vector into a likelihood measure. The lines tend to get closer to high flow parts of the hydrograph, illustrating how NSE - as mentioned in section 2,1 - allows for smaller relative errors when it comes to peak discharges. Superimposing NSE error isolines with the confidence interval of the discharge observations shows how this objective function can enforce the prediction of peak flows with a misleading accuracy: error isolines are inside the confidence interval of the discharge in this part of the hydrograph, illustrating how the objective function may detect modelling errors where the uncertainty range of the observed data is inconclusive;

- the top right window (b) displays error isolines of the Croke (2007) objective function. It shows that the model error allows for larger errors when the observations are uncertain and enforces a good mimic of the observations that are reliable. It results in a calibration that enforces really good mimic specifically when discharge observation are accurate;

- the bottom left window (c) displays the case of the Liu et al. (2009) which is quite particular, as errors span a limited range of values. A same error value is assigned to all predictions outside the confidence interval of discharge observations. Hence, the calibration is influenced mainly by the selection of behavioural simulations, depending on the percentage of predicted points inside the confidence interval of the discharge observations, rather than by the score of the objective function;

- the bottom window (d) displays error isolines of the DEC objective function in the specific case where modelling distances $\sigma_{\text {mod,i } i}$ are set to a constant. It illustrates how the DEC combines both the discharge uncertainty and the hydrologist's expertise (encapsulated in the definition of $\Omega_{\text {mod }}$ ). Error isolines run alongside the confidence interval of the hydrograph (i.e. the discharge envelop), showing that the objective function detects any modelling error inside the confidence interval. Moreover, as the modelling distance is here set to a constant, error isolines illustrate the case where the DEC enforces equally the calibration around the discharge envelop. Finally, the way to catch those discharge envelop can be adapted to model objectives by defining other modelling distance bounds.

\section{Methodology for the DEC evaluation}

\subsection{Case study: application of the DEC to flood modelling}

For the purpose of evaluation, we consider the calibration of a rainfall-runoff model dedicated to flash flood modelling. We look for the calibration and evaluation of a distributed and physicallybased model called MARINE (Modélisation de l'Anticipation du Ruissellement et des Inondations pour des évéNements Extrêmes), developped specifically for flash flood simulation. The equations describing the main flash flood processes (infiltration, overland flow, channel routing) are detailed hereafter. Low rate flow processes such as evapotranspiration, or baseflow are neglected. For more detailed information on the MARINE model, please refer to Roux et al. (2011) and Garambois et al. (2015). MARINE simulations require the calibration of six physical parameters: soil depth $C_{z}$, lateral hydraulic conductivity $C_{T O}$, hydraulic conductivity of the riverbed $C_{k r}$, saturated hydraulic conductivity $C_{k}$, and the flood plain and riverbed Manning roughness coefficients, respectively $n_{p}$ and $n_{r} . C_{Z}, C_{T O}, C_{k r}$ and $C_{k}$ are multiplicative constants of the corresponding spatialized parameters $z, T_{0} \mathrm{~K}$, and $K_{r}$.

The model is applied on the Gardon catchment at Anduze $\left(543 \mathrm{~km}^{2}\right)$. According to its physical properties (steep slope, thin soil depth) and its geographical location in the French Mediterranean area, this head watershed has a highly contrasted hydrological regime with frequent occurrences of flash floods. A set of 14 extreme events, recorded over 20 years, is considered. The hydrological model is forced with rainfall data issued by the ARAMIS radar network (Météo France, Tabary, 2007). It provides inputs with a time resolution of $5 \mathrm{~min}$ and a spatial resolution of $1 \mathrm{~km} \times 1 \mathrm{~km}$. Rainfall data is provided without uncertainty. Their calculation is a topic in its own right (Delrieu et al. (2014)), which is beyond the scope of this study.

The initial state is extracted from the SAFRAN-ISBA-MODCOU (SIM) hydro-meteorological model outputs (Habets et al., 2008). The model provides the humidity indexes of a conceptual root zone horizon. As for the rainfall input, their uncertainty is not considered.

The observed discharge data were provided by the French 


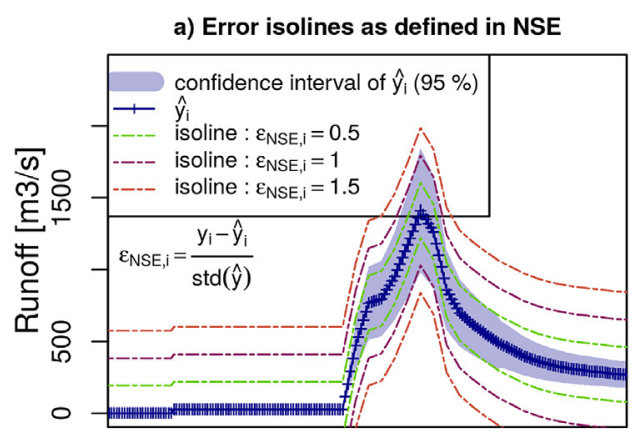

c) Error isolines as defined in Liu et al(2009)

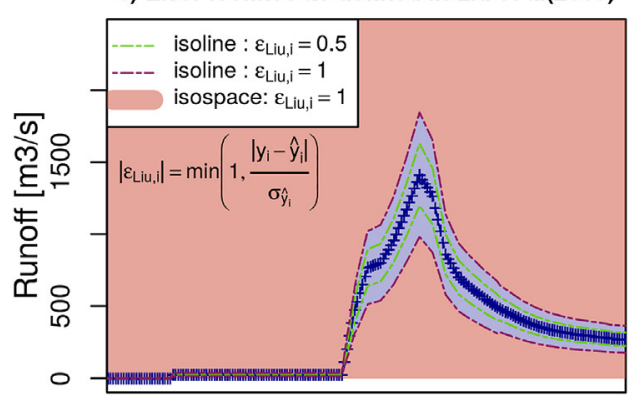

b) Error isolines as defined in Croke (2007)

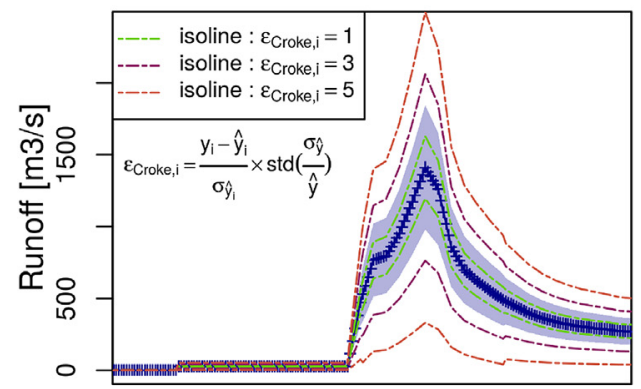

d) Error isolines as defined in DEC method

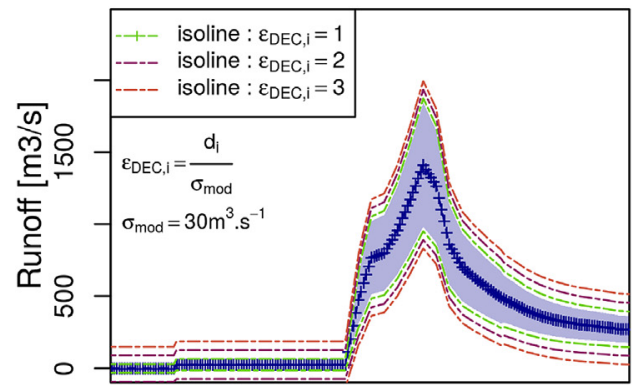

Fig. 1. Error isolines according to different model errors formulations: a) Nash-Sutcliffe efficiency (NSE); b) the Croke (2007); c) the Liu et al. (2009); d) Discharge Envelop Catching efficiency, with a constant value of the distance bounds around the discharge envelop $\left(\sigma_{\text {mod,i }}\right)$ equal to two times the catchment module ( $30 \mathrm{~m}^{3} \mathrm{~s}^{-1}$ ).

operational flood forecasting services (SCHAPI and SPC). Uncertainty discharge is evaluated from the rating curve. It is assumed that the uncertainty standard deviation $\sigma_{H}$ increases linearly with the observed stage $H$ :

$\sigma_{H}=a^{*} H+b$

with $\mathrm{a}$ and $\mathrm{b}$ depending on the gauging station characteristics at Anduze. The discharge uncertainty standard can be deduced from the stage discharge conversion $\hat{Y}=g(H)$ :

$\sigma_{\tilde{Y}}=\sigma_{H} * g^{\prime}(H)$

Finally it is assumed that the uncertainty is normally distributed. This approximation is good enough to determine confidence intervals.

\subsection{The calibration methodology using DEC objective function}

First we define the modelling distance bounds $\left(\sigma_{\text {mod, } i}\right)$. Considering the MARINE model assumptions, a coarse prediction of baseflow is expected. $\left(\sigma_{\text {mod, } i}\right)$ is set at a minimum of the catchment module $\left(Q_{\text {catchment }}\right)$. The studied events present high flow variations from a module of $15 \mathrm{~m}^{3} \mathrm{~s}^{-1}$ to peak flows reaching $1000 \mathrm{~m}^{3} \mathrm{~s}^{-1}$. Modelling distance bounds are adapted to this amplitude by setting the modelling distance bounds proportional to the observed discharge:

$\sigma_{\text {mod }, i}=Q_{\text {catchment }}+0,02 * \widehat{y}_{i}$

5000 parameter sets are extracted from an uniform distribution on bounded intervals in $\Re^{6}$. The MARINE model is run with these sets. Each resulting prediction - named $s$ - is weighted according to the DEC objective function:
$W_{D E C}^{S} \propto \exp \left(-(D E C)^{2}\right)$

where $W_{D E C}^{S}$ corresponds to the weight given to $\mathrm{s}$, according to the DEC likelihood measure. Finally for each time step, the calibration provides a distribution of weighted predictions. The median values of each distribution are considered as the average discharge prediction, while the 5th and 95th percentiles represent the bounds of discharge prediction uncertainty.

For the sake of simplicity, we designate hereafter by "DEC calibration", the calibration methodology based on the DEC objective function.

\subsection{Comparative evaluation of the DEC calibration}

Results of the DEC calibration is compared to those obtained with other methodologies. We applied the Liu et al. (2009) methodology and the Croke (2007) methodology as they both integrate discharge uncertainty into calibration. The widespread GLUE methodology is also applied (Beven and Binley, 1992), as a reference. Table 1 sums up modelling errors and weights used in each calibration methodology.

\section{Results}

\subsection{Calibration results using the DEC objective function}

Fig. 2 shows at the top window, the hydrograph simulation of six flash flood events with MARINE model after calibration using the DEC objective function. The dark blue envelop corresponds to the confidence interval of the observed discharge and the orange envelop to the confidence interval of the simulated discharge. The light blue envelop defines the region of model acceptability.

The bottom frame displays the modelling errors computed by the objective function. When the prediction lies within the discharge confidence interval (dark blue envelop), the modelling 
Table 1

Summary of the calibration methodologies: modelling error and weights.

Likelihood measures are computed on all the events.

\begin{tabular}{|c|c|c|}
\hline Method & Modelling error & Weight of the simulation $\mathrm{s}$ \\
\hline GLUE & $\varepsilon_{N S E}=\frac{y_{i}-\widehat{y_{i}}}{\operatorname{std}(\widehat{y})}$ & $W_{N S E}^{S} \propto N S E^{s}$ If $N S E^{s} \geq 0,6^{*} \max \left(N S E^{s}\right)^{\mathrm{a}} ;$ else 0 \\
\hline Liu et al. (2009) & $\left|\varepsilon_{\text {Liu }}\right|=\min \left(1,\left|\frac{y_{i}-\widehat{y_{i}}}{\sigma_{\widehat{y_{i}}}}\right|\right)$ & $W_{\text {Liu }}^{s} \propto \sum_{i} \exp \left(-\varepsilon_{\text {Liu }, i}^{2}\right)$ If $\left\{y_{i} \in\left[\widehat{y}_{i}-\sigma_{\widehat{y_{i}}} ; \widehat{y}_{i}+\sigma_{\widehat{y_{i}}}\right]\right\} \geq 85 \%^{\mathrm{b}}$; else 0 \\
\hline Croke (2007) & 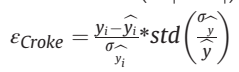 & $W_{\text {Croke }}^{s} \propto \sum_{i} \exp \left(-\varepsilon_{\text {Croke, },}^{2}\right)$ \\
\hline DEC & $\varepsilon_{D E C}=\frac{d_{i}}{\sigma_{\bmod , i}}$ & $W_{D E C C}^{S} \propto \exp \left(-\left(E_{s}^{90 t h}\right)^{2}\right)$ \\
\hline
\end{tabular}

${ }^{\text {a }}$ NSE threshold is set to $65 \%$ of the maximum NSE value obtained running all the parameter sets.

b The minimum percentage required (85\%) is set in order to select enough behavioural predictions for statistical use.
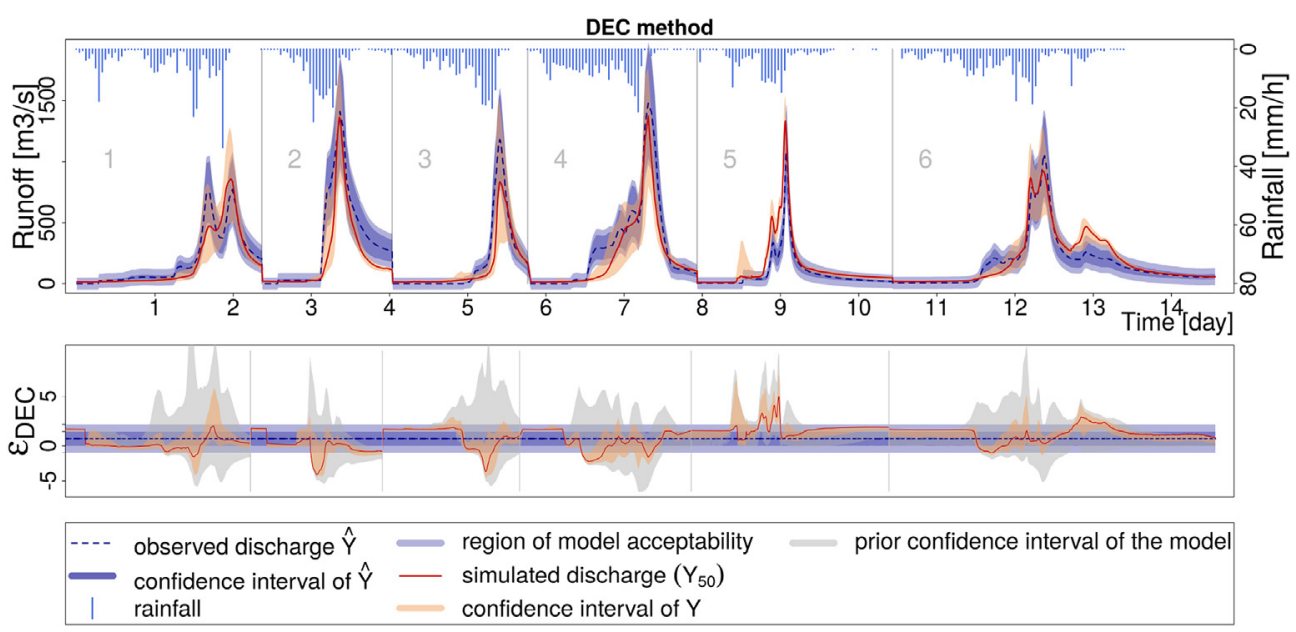

Fig. 2. Top window: Hydrograph of 6 out of 14 selected flash flood events supplied by the DEC calibration; bottom window: remaining modelling errors along the hydrograph with median prediction in red, and range of modelling errors into the confidence interval of prediction (orange). The grey envelop corresponds to the covered range of modelling errors without any selection of parameter sets. (For interpretation of the references to colour in this figure legend, the reader is referred to the web version of this article.)

error is set to zero. When the prediction is enclosed in the interval of model acceptability, modelling error is lower than 1 and it is assessed as acceptable. When the modelling error exceeds the light blue envelop, it is set to a value exceeding 1 and an error of modelling is detected. In grey are represented the confidence interval of the modelling error without any parameters sets weighting. From this display, sensitive part of the hydrograph to calibration could be detected comparing the grey and the orange envelop. As well, it emphasizes where modelling errors remain after calibration.

The hydrographs show that the observed discharge is globally well mimicked by the median prediction of the model. One flow peak is underestimated, another is overestimated, but the reproduction of the others flow peaks is close to the observed discharge. $90,11 \%$ of the median prediction points of the 14 simulated events are inside the interval of model acceptability (see Table 3). Regarding model failing, the model tends to underestimate flow peak and the rising limb. In contrast, the really early rising might be overestimated as in the events 3 and 5.

Table 2

Percentage of subsurface flow during the flood. (when $\hat{y}_{i}>150 \mathrm{~m}^{3} . \mathrm{s}-1$ ).

\begin{tabular}{lllll}
\hline (\%) & GLUE & Croke (2007) & Liu et al. (2009) & DEC \\
\hline q5 & $9,3 \%$ & $16,9 \%$ & $22,7 \%$ & $16,6 \%$ \\
q50 & $30,0 \%$ & $35,8 \%$ & $33,6 \%$ & $35,3 \%$ \\
q95 & $43,7 \%$ & $45,3 \%$ & $41,0 \%$ & $44,0 \%$ \\
\hline
\end{tabular}

Table 3

NSE on 14 flash flood events. The NSE formula is successively used to compare i) the median prediction $\mathrm{Y}_{50}^{\text {th }}$ with the discharge observation $\hat{Y}$; ii) the lower bound prediction $Y_{5}^{\text {th }}$ with the lower bound of the confidence interval of the discharge $\hat{Y}_{5}^{\text {th }}$; iii) the upper bound prediction $Y_{95}^{\mathrm{th}}$ with the upper bound of the confidence interval of the discharge $\hat{Y}_{95}^{\text {th }}$.

\begin{tabular}{lllll}
\hline & GLUE & Croke (2007) & Liu et al. (2009) & DEC \\
\hline Statistic on all data series & & & & \\
Median prediction & 0,77 & 0,63 & 0,76 & 0,76 \\
Lower bound prediction & 0,63 & 0,48 & 0,57 & 0,62 \\
Upper bound prediction & 0,80 & 0,83 & 0,82 & 0,85 \\
\hline
\end{tabular}

The event 5 stands as an exception, as the flow prediction globally overestimates the observed discharge. Parameter calibration has little impact on modelling error range during this event. Those differences may suggest more data inconsistency than modelling error, as prediction errors appear to be specific to this event.

The base flow is also well predicted as the median prediction and its confidence interval during low flows respect the interval of model acceptability. User expectations are satisfied. In fact, the baseflow prediction is quite coarse as the median prediction shows for some events a relative error of $50 \%$, but it is enough to user expectations, as defined by the DEC efficiency (ie the choice of the distance range). The calibration barely restricts the interval of prediction during low flows, as the grey and orange intervals are similar along this part of hydrograph for all events. It shows that the 
calibration is not sensitive to low flow prediction but rather to rising limb and peak flow ones. This remark is important for interpreting the parameter set weighting. Calibration results parameter sets weighting - will be informative for the related hydrological process models as they are controlled by the calibration. On the opposite, recession modelling appears not to be sensitive to the calibration but rather to model structure and input data quality.

\subsection{Comparison results for all calibration methodologies}

The comparison aims to determine to what extent the parameter selection or hydrograph reproduction depends on the calibration methodology. First the posterior distribution of the parameters is compared and prediction discrepancies are detected and explained according to objective function properties. Then the related consequences to the discrepancies on hydrograph reproductions are analyzed.

\subsubsection{Comparison of parameter posterior distributions}

Fig. 3 shows the parameter posterior distribution issued from each calibration methodology. Those distributions reflect the firstorder sensitivity of parameters to calibration methodology.

All calibration methodologies show that $C_{Z}$ and $C_{T 0}$ are sensitive parameters. It reflects how important for model performance are soil properties, both in terms of water storage capacity and subsurface flow quantification.

With the three calibration methodologies GLUE, Liu et al. (2009) and DEC, storage capacity of the model is mainly controlled by the $C_{Z}$ parameter, the infiltration parameter $C_{k}$ being not sensitive to calibration. On the opposite, the Croke (2007) method shows a sensitivity to $C_{k}$ parameter: only high values of $C_{k}$ results in behavioural simulations. It seems that the calibration does not have to limit the soil infiltration capacity, as calibration methods either allow or impose high values of infiltration velocity. Finally, all calibration methodologies suggest that runoff production in the MARINE model comes from soil storage capacity exceedence (Dunne, 1978).

The sensitivity of the transmissivity of the soil ( $C_{T 0}$ parameter $)$ results from the significant contribution to floods of subsurface flow. The proportion of subsurface flow during high part of hydrographs $\left(\hat{\boldsymbol{y}}_{\boldsymbol{i}}>150 \mathrm{~m}^{3} \mathrm{~s}^{-1}\right.$, Table 2$)$ ranges between $9 \%$ and $45 \%$, whatever the calibration method. The similar range of values for the proportion of subsurface flow does not reflect the discrepancies between posterior distributions of the $C_{T 0}$ parameter. Actually, $C_{T 0}$ posterior distributions are correlated with the $C_{Z}$ posterior ones. Discrepancies of $C_{T O}$ posterior distributions seem to compensate differences between $C_{Z}$ posterior distributions producing at the end a similar volume of subsurface flows.

Looking at the posterior distributions of roughness coefficients, which control surface flow dynamics, only the Liu et al. (2009) and the Croke (2007) methods show sensitivity to the river roughness $\left(n_{r}\right.$, Fig. 3). Considering the case of the Croke (2007) method, Fig. 4 shows that the defined error model induces sensitivity to the early rising limb of each event. Indeed, the modelling error interval of the early rising limb obtained without parameter set weighting is huge, and calibration is mainly concerned with minimizing those modelling errors. Finally, the fact that calibration is focused on the early rising limb may explain the sensitivity of the model to river roughness coefficient. Considering the Liu et al. (2009) method, as modelling errors are all valued the same falls outside the confidence interval of the observed discharge, their representation does not provide an explanation. Nevertheless, we may suppose, that the small confidence interval around the early rising limb equally makes the Liu et al. (2009) method to enforce accurate prediction for having few modelling errors in this hydrograph part.

The calibration of the last parameter - the coefficient of transmissivity of the riverbed - results in different posterior distributions between Liu et al. (2009) and the three other methodologies. Only the Liu et al. (2009) method shows sensitivity to this parameter. This sensitivity is not easy to explain as the Liu et al. (2009) methodology is not focused in any particular hydrological processes. Nevertheless, it seems that the $C_{k r}$ parameter has a compensatory effect on the selection of the other parameters, as correlations between $n_{r}$ and $C_{T 0}$ parameters appear particularly when calibrating the model with the Liu et al. (2009) methodology.

\subsubsection{Hydrograph reproduction comparison}

Fig. 5 shows the hydrographs of 6 out of 14 flash flood events outputed by the different calibration methods. Observed discharge and corresponding uncertainty are in blue, the median prediction in red and prediction uncertainty in orange. Significant systematic under- (or over-) estimation is visible. Particularly, the Croke (2007) method tends to underestimate flood discharge, for almost the presented events. On the opposite, Liu et al. (2009) method overestimates the peak discharge, giving a confidence interval of prediction exceeding that of the observed discharge.

Hydrographs show periods when the discharge confidence
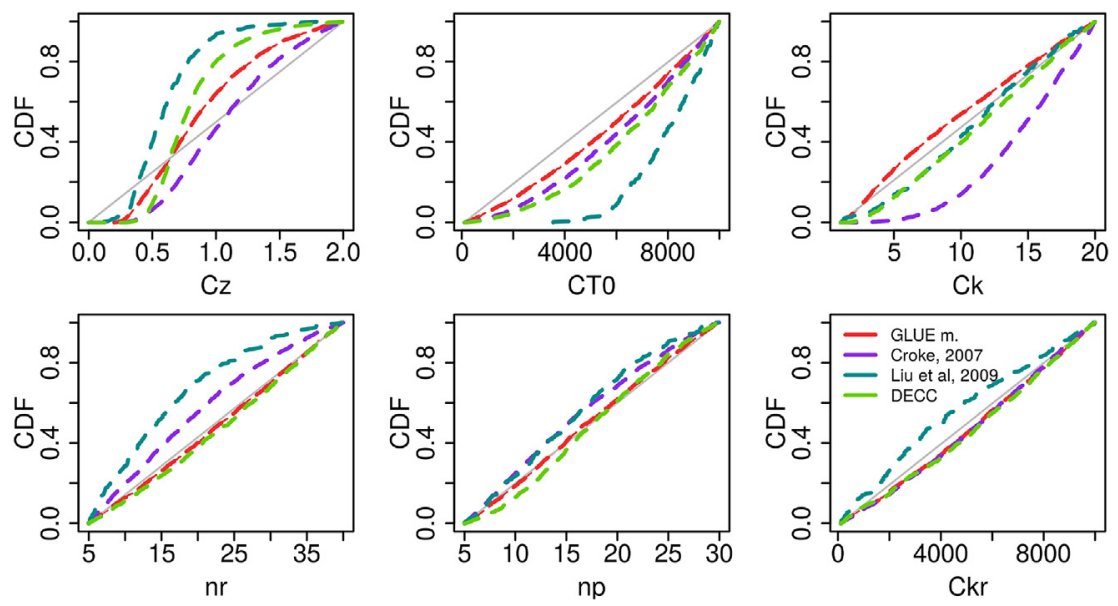

Fig. 3. Posterior distributions of parameters after calibration: soil depth $C_{z}$; lateral hydraulic conductivity $C_{T 0}$; saturated hydraulic conductivity $C_{k}$; and the riverbed and flood plain Manning roughness coefficients respectively $n_{r}$ and $n_{p}$; hydraulic conductivity of the riverbed $C_{k r}$. 

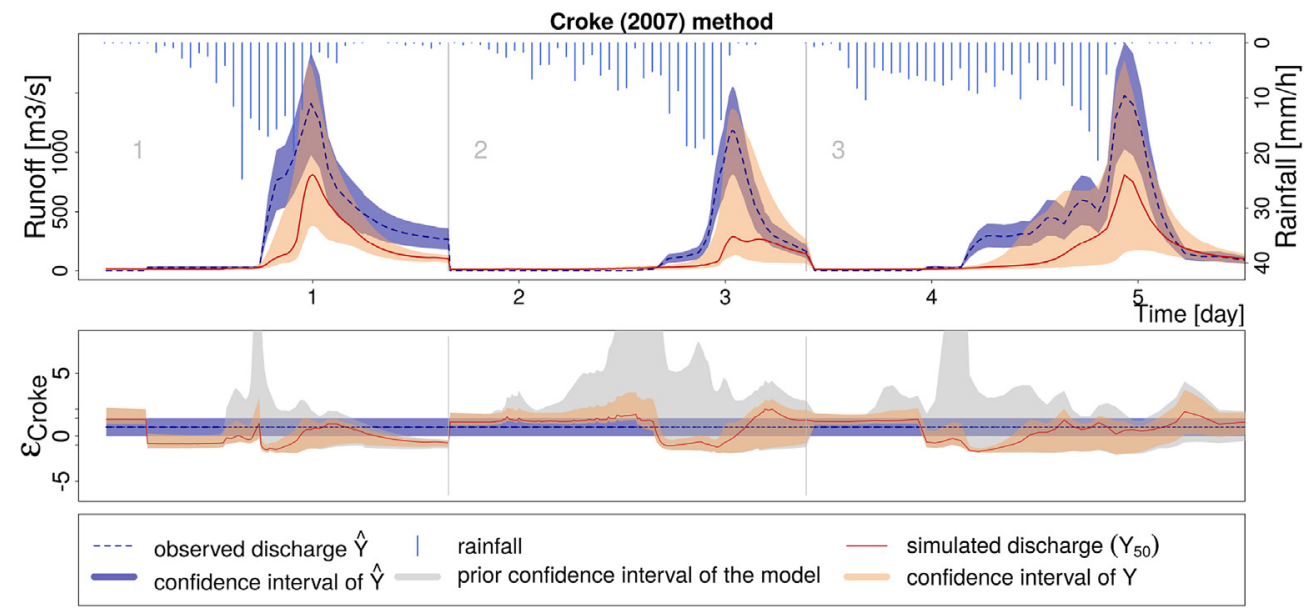

Fig. 4. Top window: Hydrographs of 3 selected flash flood events supplied by the Croke (2007) calibration; bottom window: remaining modelling errors along the hydrograph with median prediction in red, and range of modelling errors into the confidence interval of prediction. The grey envelop corresponds to the covered range of modelling errors without any selection of parameter sets. (For interpretation of the references to colour in this figure legend, the reader is referred to the web version of this article.)
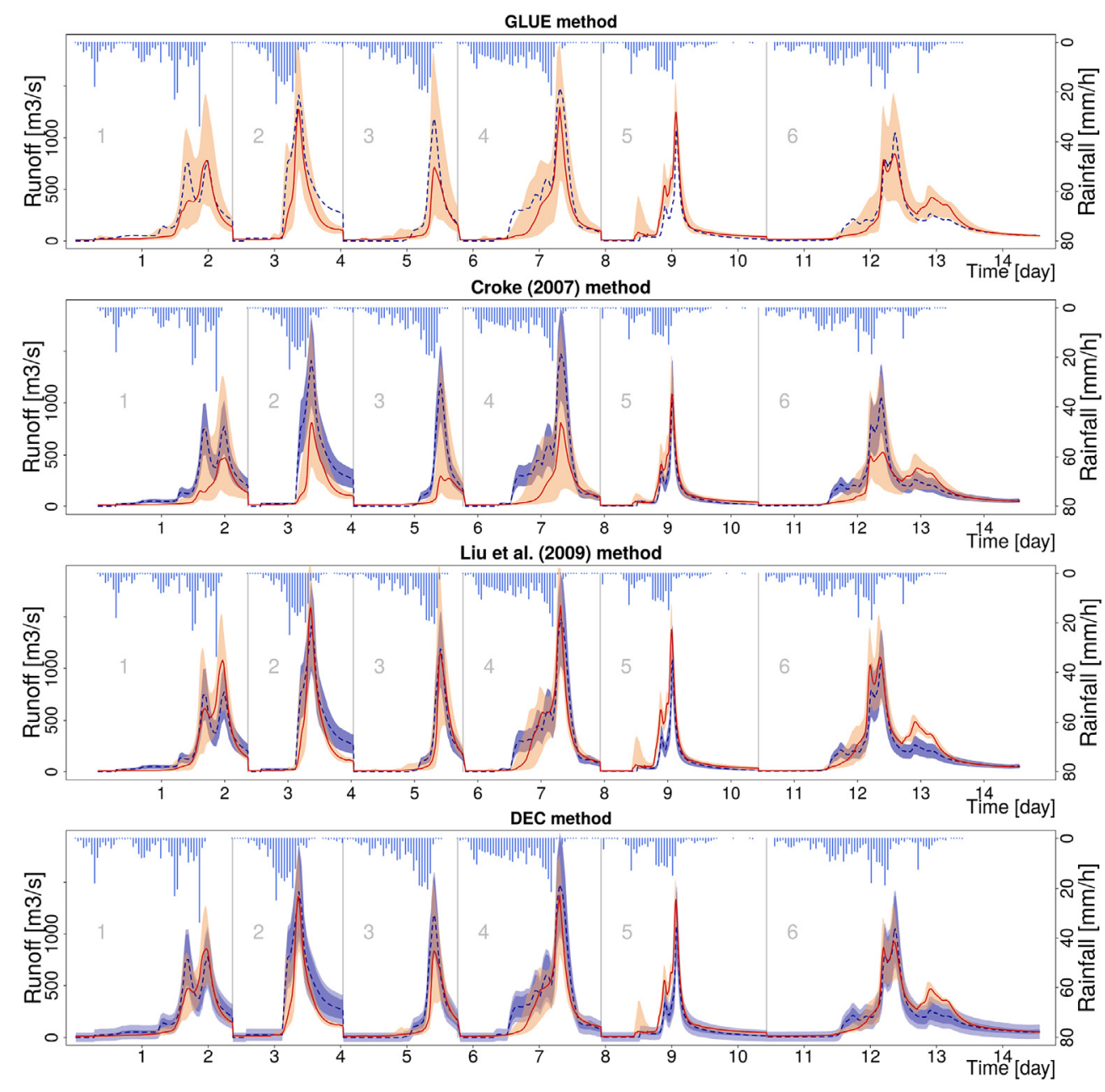

Fig. 5. Hydrograph of 6 out of $\mathbf{1 4}$ selected flash flood events (for greater clarity) supplied by the different calibration methods: a) GLUE method; b) Croke (2007); c) Liu et al. (2009) method; d) DEC method. Refer to Fig. 2 for the legend. 
interval falls outside the prediction uncertainty whatever the calibration method. Those periods could not be simulated properly by the MARINE model. Actually, it emphasizes either the weakness in the model or the input data uncertainty.

\subsubsection{Comparison of global performances}

Table 3 gives the NSE scores successively calculated between the median prediction $Y_{50}^{\text {th }}$ and the observed discharge $\hat{Y}$ (line 1); the lower bound prediction $Y_{5}^{\text {th }}$ and the lower bound of the observed discharge confidence interval $\hat{Y}_{5}^{\text {th }}$ (line 2); the upper bound prediction $Y_{\mathbf{9 5}}^{\text {th }}$ and the upper bound of the observed discharge confidence interval $\hat{Y}_{95}^{\text {th }}$ (line 3). The aim is to assess both the discharge prediction and the confidence interval of that prediction.

Representation of the observed discharge is similarly reached by the GLUE, DEC and Liu et al. (2009) methodologies, with a NSE score equal to 0,78 and 0,76 respectively. Croke (2007) has the lowest performance with a NSE score equal to 0,63 . As said before, the latter method tends to underestimate flood peak (Fig. 5). Similarly, the lower limit of the prediction is underestimated during flow peak with this method and results in the poorest score for the prediction of the lower bound (score $=0,48$ ). Finally, according to the NSE score, the GLUE, the Liu et al. (2009) and the DEC methods show similar results for the median prediction as well as for the interval bounds ones.

Considering another global index for prediction assessment, Table 4 presents the percentage of evaluated points located inside the acceptability zone defined in the DEC definition (equation (11)). The acceptability zone is defined according to user expectations, and consequently appears as the aim of the calibration. The DEC method gives the best percentage with more than $90,26 \%$ evaluated points inside the acceptability zone. GLUE method and the Liu et al. (2009) perform similarly with a score of $89,32 \%$ and $89,73 \%$, respectively. Regarding the NSE assessment, the Croke (2007) method gives the lowest result. Considering the prediction for the high parts of the hydrographs (second column, Table 4), the scores give the same range of model performance with best predictions for the DEC method, then in order the Liu et al. (2009) one, the GLUE one, and finally the Croke (2007) one.

Model prediction can alsol be assessed according to water volume flowing at catchment outlet. The bias between predicted and observed discharge reflects the predicted water balance quality. As we know that the model is not accurate for low flow prediction, we calculate the bias only for observed discharge higher than $150 \mathrm{~m}^{3} \mathrm{~s}^{-1}$ (Table 5).

Contrary to the previous metric assessments, calibration methodologies present here very contrasted results. Croke (2007) underestimates the median prediction and the lower bound prediction is far below the interval bound of the observed discharge. It is related to the fact that the method tends to underestimate peak flows as it has been already mentioned when studying hydrograph reproduction (Fig. 5). On the other hand, but not to the same degree, Liu et al. (2009) overestimates the median prediction and the lower bound prediction. The most important discrepancy is the

Table 4

Percentage of evaluated points of the median prediction inside the acceptability zone defined by in the DEC definition $(\S 3,2)$.

\begin{tabular}{lll}
\hline \multirow{2}{*}{ Method } & \multicolumn{2}{l}{ Percentage of accepted points of the median prediction } \\
\cline { 2 - 3 } & All points & Prediction of points where $\hat{y}_{i}>150 \mathrm{~m}^{3} \mathrm{~s}^{-1}$ \\
\hline DECC & $90,26 \%$ & $76,9 \%$ \\
GLUE & $89,32 \%$ & $74,1 \%$ \\
Liu et al., 2009 & $89,73 \%$ & $75,7 \%$ \\
Croke 2007 & $87,86 \%$ & $68,9 \%$ \\
\hline
\end{tabular}

Table 5

Discharge prediction bias on 14 flash flood events when observed discharged is higher than $150 \mathrm{~m}^{3} \mathrm{~s}^{-1}$. As for NSE calculation, median prediction $Y_{50}^{\text {th }}$ is compared to the observed discharge $\hat{Y}$ and the predicted bounds $\left(Y_{5}^{\text {th }}\right.$ and $\left.Y_{95}^{t h}\right)$ are compared to the bounds of the confidence interval of the discharge $\left(\hat{Y}_{5}^{\text {th }}\right.$ and $\left.\hat{Y}_{95}^{\text {th }}\right)$.

\begin{tabular}{lllll}
\hline$\left(\mathrm{m}^{3} \cdot \mathrm{s}^{-1}\right)$ & GLUE & Croke (2007) & Liu et al. (2009) & DEC \\
\hline Median prediction & -23 & -141 & 69 & 5,7 \\
Lower bound prediction & -59 & -155 & 55 & 17,1 \\
Upper bound prediction & 149 & 21 & 122 & 15,1 \\
\hline
\end{tabular}

overestimation of the upper bound prediction. Actually, hydrograph given by the Liu et al. (2009) method shows this overestimation. It may not appear in the previous score as it represents only a few points during flow peaks, therefore the contribution to the NSE score may not be significant. GLUE presents an overestimation of the upper bound and an underestimation of the lower bound, and consequently gives a larger confidence interval of prediction than the confidence interval of the observed discharge. The confidence interval bandwidth depends on the NSE threshold arbitrarily chosen in order to separate behavioural and non behavioural simulation. The choice of a higher NSE threshold may have decreased the confidence interval bandwidth and therefore resulted in more relevant prediction results.

Overall, only the DEC provides reasonable bias values. Indeed, the median prediction as well as the bound predictions have a bias that does not exceed $18.0 \mathrm{~m}^{3} \mathrm{~s}^{-1}$, which represents less than $5 \%$ of the average of the observed discharge that are higher than $150 \mathrm{~m}^{3} \mathrm{~s}^{-1}$.

In order to explain the discharge bias discrepancies, we must step back on parameter posterior distribution. Described in $\S 4,3,1$, all calibration methodologies show a model sensitivity to $C_{z}$ parameter values, but the resulted posterior distribution of this parameter differs. In particular, calibration methodologies can be ranked according to the median value of $C_{z}$ parameter posterior distribution. Liu et al. (2009) gives the lower $C_{z}^{50 t h}$ followed by, DEC, GLUE and finally Croke (2007). The selected ranking corresponds to the ranking of bias of the medium prediction, from Liu et al. (2009) method showing the highest overestimation, to the Croke (2007) method presenting the highest underestimation of the median prediction. Actually, it makes sense that the model calibrated with lower depth of storage capacity gives a higher discharge response and inversely.

Moreover, we can notice that posterior distributions of the $C_{z}$ parameter from DEC and Liu et al. (2009) reflect a more restricted range of $C_{z}$ value, than for the other methods. It may explain that these methods give smaller confidence interval of prediction around the median discharge prediction.

Finally, most of the discharge bias discrepancies between the different calibration methods may be explained as resulting from $C_{z}$, the parameter posterior distribution. The overestimation of the upper bound prediction during high flows by Liu et al., 2009 is not completely clarified. It may result either from the particular calibration of the riverbed transmissivity, $C_{k r}$ with this method, or from the selection of smaller values for $C_{z}$ parameter, that limits soil storage capacity. Further investigation should be done to confirm it.

\section{Conclusion}

We presented a calibration method that consistently integrates uncertainty of the discharge observations, model specifics and user-defined tolerance. This is achieved by introducing a new objective function called Discharge Envelop Catching efficiency (DEC). The main idea of the method is enable the end-user to define an acceptability region around the confidence interval of the 
discharge, in relevance with user's expectations. The $90^{\text {th }}$ percentile of distance distribution from prediction to the acceptability zone is used as the metric score used to assess the model. This score is considered more appropriate than the average of the distribution as it gives priority to the minimization of high distances to the acceptability region.

Using the DEC objective function, a calibration of the MARINE model is tested. The DEC method provides optimal parameter sets since high values of the NSE (0.76) are obtained with the resulting discharge prediction. Also, for $90.11 \%$ of the assessment points along the hydrograph, the discharge prediction is enclosed in the acceptability zone. This score is especially conclusive considering that input uncertainty was not taken into account.

We find that the parameter posterior distribution depends on the related calibration method, affirming the role of the objective function. Regarding the impact of the calibration on the modelling error along the hydrograph, it appears that each calibration enforces the adequacy between observed and predicted discharge at different points or parts of the hydrographs. To be relevant, the assessment of parameter posterior distribution has to be combined with the study of calibration impacts on the hydrographs. Regarding the DEC calibration method, it mainly impacts the prediction of flood rising limbs and flow peaks. The resulting parameter distribution will be most informative for flow processes occurring during the corresponding parts of the hydrographs.

Assessment with the NSE provides similar results from a calibration methodology to another, for the median prediction as well as for its confidence interval, although the DEC performs slightly better in average. The flood volume is significantly better predicted when using the DEC method. Likewise, the DEC provides a confidence interval for flood volume prediction that is more relevant with respect to the uncertainty of the discharge observations and of the related observed flood volume.

\section{References}

Beven, K., 2006. A manifesto for the equifinality thesis. J. Hydrol. 320, 18-36. http:/ dx.doi.org/10.1016/j.jhydrol.2005.07.007.

Beven, K. Binley, A., 1992. The future of distributed models: model calibration and uncertainty prediction. Hydrol. Process. 6, 279-298. http://dx.doi.org/10.1002/ hyp.3360060305.

Beven, K., Binley, A., 2014. GLUE: 20 years on. Hydrol. Process. 28, 5897-5918. http://dx.doi.org/10.1002/hyp.10082.

Coxon, G., Freer, J., Westerberg, I.K., Wagener, T., Woods, R., Smith, P.J., 2015. A nove framework for discharge uncertainty quantification applied to 500 UK gauging stations. Water Resour. Res. 51, 5531-5546. http://dx.doi.org/10.1002 2014WR016532.

Croke, B. 2007. In: Oxley, Les, Kulasiri, Don (Eds.), The Role of Uncertainty in Design of Objective Functions. International Congress on Modelling and Simulation (MODSIM 2007), vol. 7. Modelling and Simulation Society of Australia and New Zealand Inc., New Zealand, pp. 2541-2547.

Delrieu, G., Wijbrans, A., Boudevillain, B., Faure, D., Bonnifait, L., Kirstetter, P.-E., 2014. Geostatistical radar-raingauge merging: a novel method for the quantification of rain estimation accuracy. Adv. Water Resour. 71, 110-124. http://dx. doi.org/10.1016/j.advwatres.2014.06.005.

Dunne, T., 1978. Fiels studies of hillslope flow processes. Hillslope Hydrol. 227-293.

Engeland, K., Gottschalk, L., 2002. Bayesian estimation of parameters in a regional hydrological model. Hydrol. Earth Syst. Sci. 6, 883-898. http://dx.doi.org 10.5194/hess-6-883-2002.

Garambois, P.A., Roux, H., Larnier, K., Labat, D., Dartus, D., 2015. Parameter regionalization for a process-oriented distributed model dedicated to flash floods. J. Hydrol. 525, 383-399. http://dx.doi.org/10.1016/j.jhydrol.2015.03.052.

Gupta, H.V., Kling, H., Yilmaz, K.K., Martinez, G.F., 2009. Decomposition of the mean squared error and NSE performance criteria: implications for improving hydrological modelling. J. Hydrol. 377, 80-91. http://dx.doi.org/10.1016/j.jhydrol.
2009.08.003.

Habets, F., Boone, A., Champeaux, J.L., Etchevers, P., Franchisteguy, L., Leblois, E. Ledoux, E. Le Moigne, P., Martin, E. Morel, S., 2008. The Safran-Isba-Modcou hydrometeorological model applied over France. J. Geophysical Res.: Atmosphere 113, 1984-2012. http://dx.doi.org/10.1029/2007JD008548.

Hornberger, G.M., Spear, R.C., 1981. An approach to the preliminary analysis of environmental systems. J. Environ. Manag. 12 (1), 7-18.

Huard, D., Mailhot, A., 2008. Calibration of hydrological model GR2M using Bayesian uncertainty analysis. Water Resour. Res. 44, W02424. http:// dx.doi.org/10.1029/2007WR005949.

Kavetski, D., Franks, S.W., Kuczera, G., 2003. Confronting input uncertainty in environmental modelling. In: Duan, Q., Gupta, H.V., Sorooshian, S., Rousseau, A.N., Turcotte, R. (Eds.), Calibration of Watershed Models. American Geophysical Union, Washington, D. C.. http://dx.doi.org/10.1029/WS006p0049

Kavetski, D., Kuczera, G., Franks, S.W., 2006. Bayesian analysis of input uncertainty in hydrological modeling: 1. Theory. Water Resour. Res. 42 http://dx.doi.org/ 10.1029/2005WR004368.

Kirstetter, P.-E., Delrieu, G., Boudevillain, B., Obled, C., 2010. Toward an error model for radar quantitative precipitation estimation in the Cévennes-Vivarais region, France. J. Hydrol. 394, 28-41. http://dx.doi.org/10.1016/j.jhydrol.2010.01.009.

Kuczera, G., 1983. Improved parameter inference in catchment models: 1. Evaluating parameter uncertainty. Water Resour. Res. 19, 1151-1162. http:// dx.doi.org/10.1029/WR019i005p01151.

Kuczera, G., Kavetski, D., Franks, S., Thyer, M., 2006. Towards a Bayesian total error analysis of conceptual rainfall-runoff models: characterising model error using storm-dependent parameters. J. Hydrol. 331 (1-2), 161-177. http://dx.doi.org/ 10.1016/j.jhydrol.2006.05.010.

Le Coz, J., Renard, B., Bonnifait, L., Branger, F., Le Boursicaud, R., 2014. Combining hydraulic knowledge and uncertain gaugings in the estimation of hydrometric rating curves: a Bayesian approach. J. Hydrol. 509, 573-587. http://dx.doi.org/ 10.1016/j.jhydrol.2013.11.016.

Liu, Y., Freer, J., Beven, K., Matgen, P., 2009. Towards a limits of acceptability approach to the calibration of hydrological models: extending observation error. J. Hydrol. 367, 93-103. http://dx.doi.org/10.1016/j.jhydrol.2009.01.016.

McMillan, H.K., Westerberg, I.K., 2015. Rating curve estimation under epistemic uncertainty. Hydrol. Process. 29, 1873-1882. http://dx.doi.org/10.1002/ hyp.10419.

Moussa, R., 2010. When monstrosity can be beautiful while normality can be ugly: assessing the performance of event-based flood models. Hydrological Sci. J. 55 (6), 1074-1084. http://dx.doi.org/10.1080/02626667.2010.505893.

Nash, J.E., Sutcliffe, J.V., 1970. River flow forecasting through conceptual models Part I: a discussion of principles. J. Hydrol. 10, 282-290. http://dx.doi.org/10.1016/ 0022-1694(70)90255-6.

Peña-Arancibia, J.L., Yongqiang, Z., Pagendam, D.E., Viney, N.R., Lerat, J., van Dijk, A.I.J.M., Vaze, J., Frost, A.J., 2015. Streamflow rating uncertainty: characterisation and impacts on model calibration and performance. Environ. Model. Softw. 63, 32-44. http://dx.doi.org/10.1016/j.envsoft.2014.09.011.

Roux, H., Labat, D., Garambois, P.-A., Maubourguet, M.-M., Chorda, J., Dartus, D., 2011. A physically-based parsimonious hydrological model for flash floods in Mediterranean catchments. Nat. Hazards Earth Syst. Sci. 11, 2567-2582. http:/ dx.doi.org/10.5194/nhess-11-2567-2011.

Schaefli, B., Gupta, H.V., 2007. Do Nash values have value? Hydrological Process. John Wiley Sons, Ltd. 21, 2075-2080. http://dx.doi.org/10.1002/hyp.6825.

Schaefli, B., Hingray, B., Niggli, M., Musy, A., 2005. A conceptual glacio-hydrological model for high mountainous catchments. Hydrol. Earth Syst. Sci. 9, 95-109. http://dx.doi.org/10.5194/hess-9-95-2005.

Schoups, G., Vrugt, J.A., 2010. A formal likelihood function for parameter and predictive inference of hydrologic models with correlated, heteroscedastic, and non-Gaussian errors. Water Resour. Res. 46 http://dx.doi.org/10.1029/ 2009WR008933.

Seibert, J., 2001. On the need for benchmarks in hydrological modelling. Hydrological Process. John Wiley Sons, Ltd. 15, 1063-1064. http://dx.doi.org/10.1002/ hyp. 446 .

Tabary, P., 2007. The new French operational radar rainfall product. Part I: methodology. Weather Forecast. 22, 393-408. http://dx.doi.org/10.1175/WAF1004.1.

Thyer, M., Renard, B., Kavetski, D., Kuczera, G., Franks, S.W., Srikanthan, S., 2009. Critical evaluation of parameter consistency and predictive uncertainty in hydrological modeling: a case study using Bayesian total error analysis. Water Resour. Res. 45, W00B14. http://dx.doi.org/10.1029/2008WR006825.

Villarini, G., Krajewski, W., 2010. Review of the different sources of uncertainty in single polarization radar-based estimates of rainfall. Surv. Geophys. 31, 100-129. http://dx.doi.org/10.1007/s10712-009-9079-x.

Vrugt, J.A., Sadegh, M., 2013. Toward diagnostic model calibration and evaluation: approximate Bayesian computation. Water Resour. Res. 49, 4335-4345. http:// dx.doi.org/10.1002/wrcr.20354. 